$\begin{array}{lllllllll}A & R & T & I & C & U & L & O\end{array}$

\title{
Festival Ver Ciencia Caribe: Un Modelo de Apropiación Social del Conocimiento Científico Regional*
}

\author{
Por: Carlos Ospina Bozzi
}

\section{RESUMEN}

El Festival Ver Ciencia Caribe se aborda desde la recuperación y valorización de la investigación científica regional, la inteligencia colectiva y la identidad cultural. Promueve el objetivo social de construir una cultura científica humanística que la población juvenil pueda llegar a sentir como propia.

El modelo de apropiación que estamos presentando a la comunidad internacional entiende que es posible producir materiales de divulgación científica, popularización y apropiación social del conocimiento, útiles tanto para el ámbito de la enseñanza formal como para el de la educación informal, mediante múltiples usos, tanto en la emisión en medios de comunicación masiva, como en el aula de clase y en otros ámbitos sociales.

\section{Mensaje con Propósito}

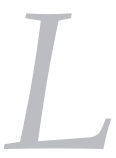

a propuesta del proyecto Ver Ciencia Caribe, gestado por la Universidad de Cartagena, se fundamenta esencialmente en la idea de articular y acuñar un concepto integrado de tres aproximaciones a la producción audiovisual, que denominamos Televisión Educativa, Científica y Cultural, en el que se diluyen las fronteras y se reconoce que la televisión científica, más allá de producir divulgación, de llevar información, o de entretener, debe ser un instrumento social al servicio del mejoramiento de la calidad de vida de nuestras poblaciones.

* Ponencia presentada como Conferencia Magistral de inauguración del Festival MIFSCIENCES, La Habana, Cuba, Junio 6 de 2006. Texto adaptado para la Revista Palobra.
Por tanto, ha de ser una televisión con un fuerte componente didáctico y cultural, de manera tal que llegue a las audiencias con un claro sentido de aplicación en sus vidas personales, en sus cotidianidades. Sólo así lograremos un verdadero diálogo entre ciencia y sociedad, particularmente cuando las inmensas mayorías de nuestros países viven bajo condiciones de pobreza económica. En su mediación pedagógica para la educación popular, el aprendizaje sólo se da cuando adquiere sentido, y el hogar del sentido está en la vida cotidiana. La juventud es el principal 
recurso para el desarrollo, y es por ello que son los jóvenes los protagonistas de nuestros esfuerzos, es a ellos a quienes invitamos a asumir el reto de constituirse en una generación de investigadores, construyendo una cultura científica con un sentido esencialmente humano, que nos conduzca al cambio.

En principio, podemos definirlo como un proyecto estratégico de comunicación social dirigido a la apropiación social del conocimiento científico regional, por parte de la juventud del Caribe colombiano, una región que alberga el 20\% de la población del país, con cerca de 7 millones de habitantes, y presenta uno de los indicadores más atrasados a nivel nacional.

En el año 2006 se implementó la experiencia piloto. Básicamente, durante tres mañanas, del 17 al 19 de mayo pasado, cerca de cinco mil jóvenes estuvieron pensando simultáneamente el futuro regional, mediante la recepción en sus colegios, de una serie de programas televisivos y la realización de unos talleres apoyados con un periódico juvenil y un manual para los orientadores.

Los jóvenes aprendieron y discutieron sobre el fenómeno de la globalización, y la relación que tiene la investigación científica y el diálogo entre la ciencia y la sociedad, con el reto de insertarnos exitosamente en el orden internacional del siglo XXI, lo cual es tarea de esta generación.

El Festival Ver Ciencia Caribe se aborda desde la recuperación y valorización de la investigación científica regional, la inteligencia colectiva y la identidad cultural. Promueve el objetivo social de construir una cultura científica humanística que la población juvenil pueda llegar a sentir como propia.

Entre sus propósitos de comunicación están la promoción del pensamiento creativo y crítico entre la juventud, generando una reflexión integral en torno al presente y futuro de la región en el marco de la globalización; popularizar el conocimiento existente sobre la realidad regional, a partir de los estudios impulsados por diversas entidades y centros de investigación de la región; acercar al público juvenil a la ciencia a través del documental científico; adelantar una indagación en torno a nuevas estrategias pedagógicas para el fomento de la cultura científica y la popularización del conocimiento; y dar continuidad al proceso de investigación sobre nuevos lenguajes de televisión educativa, científica y cultural iniciado en la Universidad de Cartagena en 1991. 


\section{Caracterización del Modelo}

Una de las más importantes cualidades de este modelo, es su carácter asociativo: dos universidades, una pública y una privada, un centro de estudios regionales, el canal regional de televisión, un prestigioso periódico y la entidad nacional de fomento a la ciencia y la tecnología.

Una segunda característica que nos interesa resaltar es su carácter participativo, que involucra a los jóvenes en la misma producción de los mensajes audiovisuales y los hace actores vivos de la construcción de una cultura científica regional, mediante la elaboración de talleres propositivos apoyados por materiales impresos.

Por ello, la tercera característica relevante del modelo es su carácter multimediado, involucrando un complejo diseño de medios y estrategias de acción para el logro de sus propósitos y objetivos.

Una cuarta característica de interés es la concepción estratégica, según la cual lo que importa es generar procesos de comunicación social, más que generar productos de comunicación social; ello se logrará mediante la participación activa de las poblaciones objeto y la conformación paulatina de una colección audiovisual de apropiación social del conocimiento científico regional.

Como quinta característica, el modelo genera espacios de investigación, promoviendo la construcción de procesos orientados hacia un abordaje investigativo en temáticas relacionadas con la comunicación de la ciencia, la educación científica, la percepción de la ciencia y la tecnología y la participación pública.

\section{El Manejo de la Información Científica}

Es en este contexto donde conviene situar el estudio de la divulgación científica a través del discurso audiovisual, desde al análisis de los mecanismos narrativos del documental de divulgación científica, haciendo uso de modelos capaces de presentar la información de una forma que resulte inteligible e interesante para el público.

En cuanto al tratamiento de la información, el modelo define cuatro criterios básicos: un criterio participativo, un criterio de lenguaje juvenil, un criterio pedagógico y un criterio estético. Así mismo, el modelo combina cuatro tipos de tratamiento: científico, periodístico, musical y lúdico. 
¿Por qué es un modelo innovador? La comunicación de la ciencia con el público busca el objetivo central de la alfabetización científica y tecnológica de los ciudadanos, desde una perspectiva crítica de interpretación de la realidad, en los niveles analítico, argumentativo y propositivo y en los ámbitos de educación formal, no formal e informal. El proyecto Ver Ciencia Caribe integra estos seis niveles y ámbitos.

Así mismo, la iniciativa incorpora el componente reflexivo, crítico y de participación pública mediante un proceso de apropiación social del conocimiento científico regional. Ello se logra involucrando a la misma población objeto de intervención, en la producción de mensajes y propuestas.

De otra parte, Ver Ciencia Caribe ofrecerá al sistema de educación secundaria y superior una importante herramienta de divulgación del conocimiento científico regional, de la que hasta el momento adolece. Esto se logrará mediante la conformación y oferta de una colección audiovisual de apropiación del conocimiento científico regional, de fácil acceso para las instituciones educativas, que tendrán la posibilidad de manejar los paquetes audiovisuales en el aula.

\section{El Abordaje Multicultural: La Relación Ciencia-cultura}

Estos procesos de indagación, que convierten a nuestras universidades en verdaderos laboratorios de investigación en el lenguaje audiovisual para la apropiación social del conocimiento, deben ser asumidos desde la comprensión de la época y el reconocimiento de la multiculturalidad. Ya no podemos hablar de ciencia local, regional o nacional, omitiendo los efectos de la transnacionalización de la cultura y su desterritorialización. Es por ello que el proyecto Ver Ciencia Caribe está fundado en un principio conceptual comprensión de la realidad regional en el marco de la comprensión de la globalización. En la primera versión de esta experiencia de comunicación

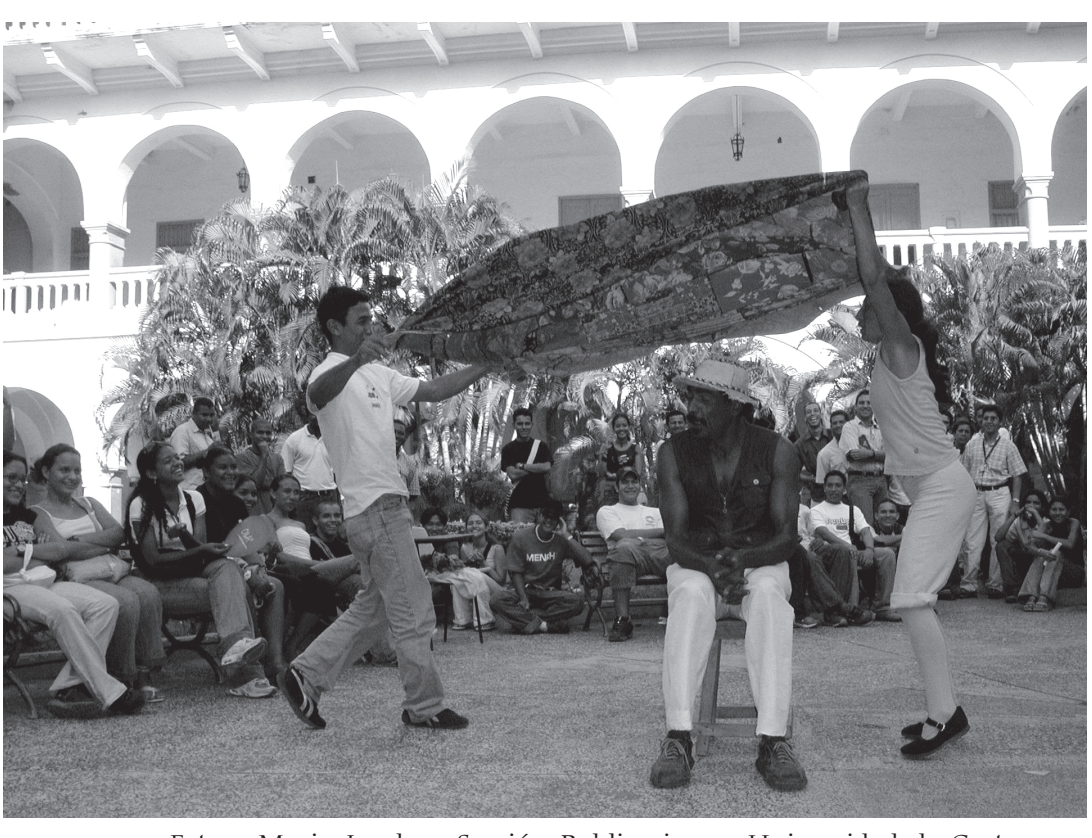

Foto: Mario Lorduy. Sección Publicaciones. Universidad de Cartagena 
1 ACEVEDO, Elsa

Beatriz, Ciencia y

Público. Tomado de

Internet. social, se propuso a la juventud la tarea de comprender la globalización como fenómeno, así como la importancia del diálogo ciencia-sociedad y la investigación científica para afrontar exitosamente los retos de siglo XXI, pues una sociedad que no se conoce a sí misma, está condenada al estancamiento. Por tanto, esta versión gira conceptualmente en torno a estos cuatro conceptos: la globalización, ciencia y conectividad, el pensamiento crítico y la creatividad.

Si bien el fenómeno de la globalización propone un proyecto homogeneizante, él mismo nos enfrenta al reconocimiento de la diferencia y la multiculturalidad. Por lo tanto, la divulgación científica y la apropiación social del conocimiento han de darse no sólo desde los polos del poder propios de la sociedad del conocimiento, sino desde las especificidades culturales, y esto es posible a partir de la generación de proyectos de comunicación que busquen un equilibrio entre lo extrínseco y lo intrínseco de las realidades regionales y locales.

Para ello, se enfatiza en la relación entre ciencia y cultura, de manera tal que la apropiación social del conocimiento se alimente de investigaciones útiles y socialmente relevantes que se dan a conocer mediante lenguajes con sentido para las audiencias específicas. Quizás una limitación de esta aproximación es la pérdida de un carácter universal de los productos, pues se habla "de tu a tu" con estas poblaciones y se abordan sus propias expresiones, desde sus especificidades culturales.

La divulgación de la ciencia se convierte en parte sustancial de la necesaria democratización del conocimiento en las sociedades modernas. Es obvio que la ciencia y la tecnología están vinculadas con todas las actividades de la vida moderna y por tanto han de estar relacionadas con las políticas de crecimiento económico y mejoramiento de la calidad de vida de la población.

Se requiere de una mayor actitud científica por parte de la gente común y una mayor participación activa en las preocupaciones de la vida cotidiana por parte de los científicos. La divulgación de la ciencia puede ayudar mucho a este acercamiento especialmente si se realiza como un proceso de comunicación que contenga conocimientos socialmente útiles. Afirma Elsa Beatriz Acevedo: "El receptor no está únicamente interesado en conocer la verdad sobre la cuestión de la que se le informa, sino también en saber cuál es el significado que esa información puede tener para él y qué consecuencias prácticas pueden derivarse para su vida cotidiana" ${ }^{1}$

El Festival Ver Ciencia Caribe responde a la idea de lograr que la ciencia deje de 
generar resistencia en el público, transformando la misma en parte sustancial de la vida cotidiana". Así mismo, se insiste en la necesidad de ubicarnos en el contexto global a partir del reconocimiento de una autoestima nacional, mediante la generación de espacios comunicativos en los cuales la divulgación científica convoque a la población a la construcción de una sociedad del conocimiento, haciendo ciencia y divulgación científica con alto grado de contenido social.

Juan José Plata es claro al afirmar que una sociedad que no es capaz de generar el conocimiento sobre sí misma y el saber hacer de sus instituciones está condenada a desaparecer. En términos de conocimiento -continúa Plata- este reto se expresa en la ampliación de las formas de circulación y uso de los conocimientos. Su propuesta de conectividad surge de la crítica a los sistemas de información que no afectan la vida ni las instituciones, ni inciden sobre lo que le sucede en el día a día a las comunidades. ${ }^{2}$ Este autor urge a construir redes de conocimiento más fuertes, dado que "no nos consumimos unos a otros, no nos leemos, no nos comunicamos". Conectividad no es más que estar dispuestos a entrar en contacto con alguien; y una cultura no es más que las redes de comunicación en las que vivimos y donde construimos una inteligencia social.

El Festival Ver Ciencia Caribe busca romper los mitos sobre la ciencia y sobre los científicos, como fuerzas extrañas, ajenas y casi sagradas, entre otras formas reconociendo que el conocimiento científico no es infalible, y está sometido a las leyes del error y el acierto. Acevedo complementa estas ideas al expresar que "de todas formas, la sociedad debe conocer, comprender y ver en la ciencia y en las innovaciones tecnológicas procesos sociales que pueden mejorar sostenidamente sus condiciones de vida. De ahí la importancia de su apropiación, mediante una divulgación, seria, sencilla, veraz y objetiva, que propicie la formación de una mentalidad nueva". ${ }^{3}$

El Festival Ver Ciencia Caribe propone un puente entre la posición de quienes consideran la divulgación científica como una actividad comunicativa que se propone transmitir al público en general conocimientos apoyados en la ciencia, que no necesariamente son científicos, y quienes consideran que la divulgación científica es únicamente aquella que se lleva a cabo fuera de la enseñanza oficial o de enseñanzas equivalentes, sin que forme parte tampoco del proceso de formación de especialistas. El modelo de apropiación que estamos presentando a la comunidad internacional entiende que es posible producir materiales de divulgación científica, popularización y apropiación social del conocimiento, útiles tanto para el ámbito de la enseñanza formal como para el de la educación

2 PLATA, Juan José, Políticas Públicas conectividad y gestión del conocimiento para la vida. Tomado de Internet.

3 Op.Cit. ACEVEDO. 
${ }^{4}$ PLATA, Op.Cit.

5 LEON, Bienvenido (1999). El Documental de Divulgación Científica, Paidos, Papeles de Comunicación 24, Barcelona, p. 43.

6 OSPINA BOZZI, Carlos. Documento de diseño del Programa Regional de Divulgación sobre el Caribe Colombiano, ORIGENES, 1991.

${ }^{7}$ OSPINA BOZZI, Carlos

y MEJIA, Silvia. Sincronía en el Espacio Tiempo. "Memorias de la Bienal Iberoamericana de la Com un i c a c i ón : Sociedad, Información y Conocimiento". Red Latina de Comunicación Social., p.p. 251-256. Universidad de El Salvador, 2003.

${ }^{8}$ Ibid, p. 134 informal, mediante múltiples usos, tanto en la emisión en medios de comunicación masiva, como en el aula de clase y en otros ámbitos sociales.

Hay que trascender el plano de la información y meternos en el plano de la formación. En palabras de Acevedo, mientras que la información contiene un margen de especulación, la formación exige un nivel de conocimiento acertado, que permite argumentar, sustentar, discutir, disentir acerca de aspectos tecnocientíficos, facilitando la reflexión crítica sobre los efectos de la ciencia y la tecnología, e incluyendo un sentido de alerta sobre posibles impactos negativos.

COLCIENCIAS, la entidad estatal de fomento a la ciencia y la tecnología en Colombia, se ha planteado las preguntas de icómo hacer más densas las redes de conocimiento pero a la vez cómo construir lazos y mecanismos de comunicación más fluidos entre las redes de generadores de conocimiento y las redes de consumidores de dichos conocimientos? ${ }^{4}$

El divulgador es más un creador que un traductor, sobre todo en el caso de la televisión, que se dirige a la experiencia cotidiana, realizando la mediación entre el discurso especializado y el general, o sea entre la ciencia y el sentido común.

De lo que se trata es de resolver "de qué forma se realiza la mediación entre las dos esferas de conocimiento -científico y cotidiano-, a través de una serie de recursos o técnicas", ${ }^{5}$ entre los cuales se destacan, por ejemplo, el uso de estructuras dramáticas y mitos.

En este sentido, nuestra tradición investigativa en materia de apropiación social se centra en la fortaleza de la cultura, con una filosofía eminentemente participativa desde el lema "la gente le habla a la gente". ${ }^{6}$ El modelo Ver Ciencia Caribe es un desarrollo de este proceso investigativo que cumple ya 16 años.

Así bien, expresa Ospina Bozzi, "más allá de la emisión televisiva, deben interesar los procesos de comunicación social que la producción misma genera al interior de la comunidades y los grupos participantes",7 produciendo fenómenos de empoderamiento que dan fe de la importancia de establecer una comunicación horizontal en un medio masivo que se ha caracterizado por su verticalidad. De esta forma, la apertura a la participación permite que "los grupos de la población trabajen activamente en la concepción y producción de los mensajes televisivos, haciendo uso de lenguajes propios de su cultura, y retomando por ejemplo elementos del humor, la música y lo coloquial". ${ }^{8}$ 
Finalmente, cuando se trata de abordar una reflexión crítica frente al papel e impacto del desarrollo científico y tecnológico sobre la vida humana y sus formas de organización social, no podemos dejar de lado el dilema político y ético de la divulgación científica.

En efecto, los desarrollos tecnológicos y las posteriores revoluciones culturales de la comunicación de masas y de la información, han generado lo que algunos autores denominan un verdadero cambio civilizatorio que ha transformado radicalmente el concepto de espacio-tiempo y por ende nos conduce vertiginosamente hacia una sociedad planetaria caracterizada por su esencia de virtualidad y su pronóstico de transformarnos en seres bio-trónicos apoyados por máquinas inteligentes de las que seremos cada vez más dependientes.

La dimensión ética adquiere entonces un valor de prioridad que aún no nos atrevemos a abordar plenamente. La propuesta de investigación de la Universidad de Cartagena en materia de comunicación para el desarrollo y de televisión educativa, científica y cultural, hace énfasis en la necesidad de abordar estas cuestiones, dejando de lado los prejuicios míticos sobre la neutralidad de la ciencia y su exclusivo impacto positivo sustentado en premisas de modernización y de modernidad.

De otra parte, esta dimensión ética sugiere promover una reflexión en torno a la ciencia y la tecnología estrechamente ligada al acontecer social y al contexto de cada región y cada país, en el caso colombiano fuertemente afectados por la insensibilidad de los grupos dirigentes frente a las condiciones de pobreza y miseria de la mayoría de la población, así como por la corrupción y los fenómenos de violencia.

El Festival Ver Ciencia Caribe propone la exploración de códigos de "explosión de sentido" fundados en el elemento cultural y sus derivaciones lúdicas, al invitar a grupos de la población a ser actores vivos del proceso comunicativo. En el caso de la serie producida para esta versión piloto, a partir de unos insumos conceptuales, un grupo de jóvenes compuso y produjo mensajes musicales en torno a las temáticas centrales del Festival, a saber, la globalización, la ciencia y la conectividad, el pensamiento crítico y la creatividad, actuando además como presentadores de la serie dirigida a la juventud del Caribe colombiano.

En síntesis, el paradigma cultural que alienta la experiencia de la Universidad de Cartagena con la construcción del modelo de apropiación social del conocimiento científico regional denominado Ver Ciencia Caribe, se resume en el uso de la tecnología audiovisual al servicio de la gente, "como una concepción derivada 
${ }^{9}$ OSPINA BOZZI, Carlos MEJIA, Silvia. Sincronía en el Espacio Tiempo. Universidad de Cartagena, Facultad de Ciencias Sociales y Educación, Palobra No, 4, Agosto de 2003, p. 139. de la reflexión en torno a la revolución tecnológica que está transformando profundamente al ser humano, sus maneras de relacionarse con los demás y ante todo consigo mismo". ${ }^{9}$

Nos identificamos filosóficamente con una ciencia y una tecnología que en lugar de cosificar al ser humano, sirvan como verdaderos instrumentos al servicio de la permanencia y el respeto de la vida en el planeta: en otras palabras, la idea de que el hombre no debe equívocamente ser ni un esclavo de las deshumanizantes maquinarias de poder, ni un esclavo tecnológico de la máquina, sino que ella ha de ser puesta -por medio de una contra-revolución cultural- al servicio de su espíritu y del rescate de la dignidad humana.

Ver Ciencia Caribe adhiere a las ideas de una ciencia con sentido, una ciencia con conciencia, y una ciencia al servicio de la gente, particularmente de los grupos más vulnerables.

El mundo reacciona ante la aldea global con un esfuerzo por reconocer los vínculos que aún lo atan a la aldea local, y a sus propias riquezas que parecen estar siendo lentamente arrebatadas por nefastos poderes que buscan instaurar un reino tecnológico, en el que es el hombre quien está a su servicio, y no ella -la tecnología- al servicio del espíritu que anima la vida.

He ahí el mayor y más grande riesgo de arrodillarnos ingenuamente ante el mito científico tecnológico de una ambigua promesa de bienestar del ser humano, relacionada con la falsa concepción de que la felicidad de los pueblos está en su capacidad de consumo. De hecho, ella no es más que una quimera, pues si todos los habitantes del planeta alcanzaran los niveles de consumo de las sociedades más avanzadas, en pocos días tendríamos que salir a las calles con máscaras de oxígeno.

La pregunta que debemos hacernos es dónde y al servicio de quién nos ubicamos, y donde sea que lo hagamos, es una opción de cada ser: a quién servimos, ¿a los poderes tecno-económicos que buscan desalojar el espíritu del planeta tierra y sólo ven la ciencia y la tecnología con fines utilitarios, como la empresa más millonaria y lucrativa de la historia? ¿A los opresivos poderes religiosos y ahora tecno-religiosos que buscan controlar la vida humana mediante el miedo y la culpa? ¿O al espíritu del amor, cuya empresa es precisamente rescatar el planeta como un laboratorio de conciencia donde podamos construir la anhelada civilización humana en la que la guerra, la explotación, la miseria, la inequidad, el egoísmo, la cosificación del ser humano y toda forma de opresión hayan sido desalojadas por siempre de la faz de la Tierra? 
Nuestra posición es clara, y es por ello que hemos accedido a este foro: Nos dirigimos hacia un mundo en el que la realidad virtual -aquella en la que la imitación de la realidad es tan perfecta que nos hace creer que ella es la misma realidad- transformará radicalmente nuestras maneras de relacionarnos, de comunicarnos, de amistarnos, y hasta de amarnos. Un mundo en que la palabra «espíritu» será anacrónica y desconocida por sus habitantes.

Nos dirigimos hacia una sociedad planetaria bio-robotrónica en la que los seres humanos irán intercambiando sus partes inutilizadas por el uso, hasta ser prácticamente convertidos en humanoides que ya no reconocen en sí su esencia, quienes interactuarán normalmente con máquinas inteligentes cada vez más capaces de respuesta autónoma, y seres clonados en serie, todos ellos esclavos de un principio que ubica la felicidad en factores externos, y no en el ser interior que aguarda en cada uno de nosotros el despertar de nuestra memoria universal.

Los descubrimientos de la física cuántica, esa nueva ciencia de la probabilidad, se acercan mucho a las antiguas filosofías, como el budismo, que plantean que la realidad física no es más que un tejido de ilusión, cuando se está descubriendo en el laboratorio de ciencia que en realidad la materia no existe, pues las partículas y subpartículas atómicas no son otra cosa que energía que aparece y desaparece constantemente, creando la apariencia de materia y de separación de la unidad de la que hacemos parte. Aunque para muchos aún sea un hueso muy duro de roer, estamos ad-portas de conocimientos hasta ahora inimaginados: la tierra no era plana, ni era el sol el centro del universo; aún más grande es la ruptura paradigmática que se nos avecina desde el seno mismo de la ciencia pura isomos energía inteligente en acción y lo que vemos es lo que manifestamos, creando infiernos y purgatorios, y por tanto podemos cambiar la película, y manifestar un mundo mejor!

Está surgiendo una nueva cultura existente en el planeta, por medio de la cual, mediante el rescate de la conciencia, el desarrollo del conocimiento y de las máquinas

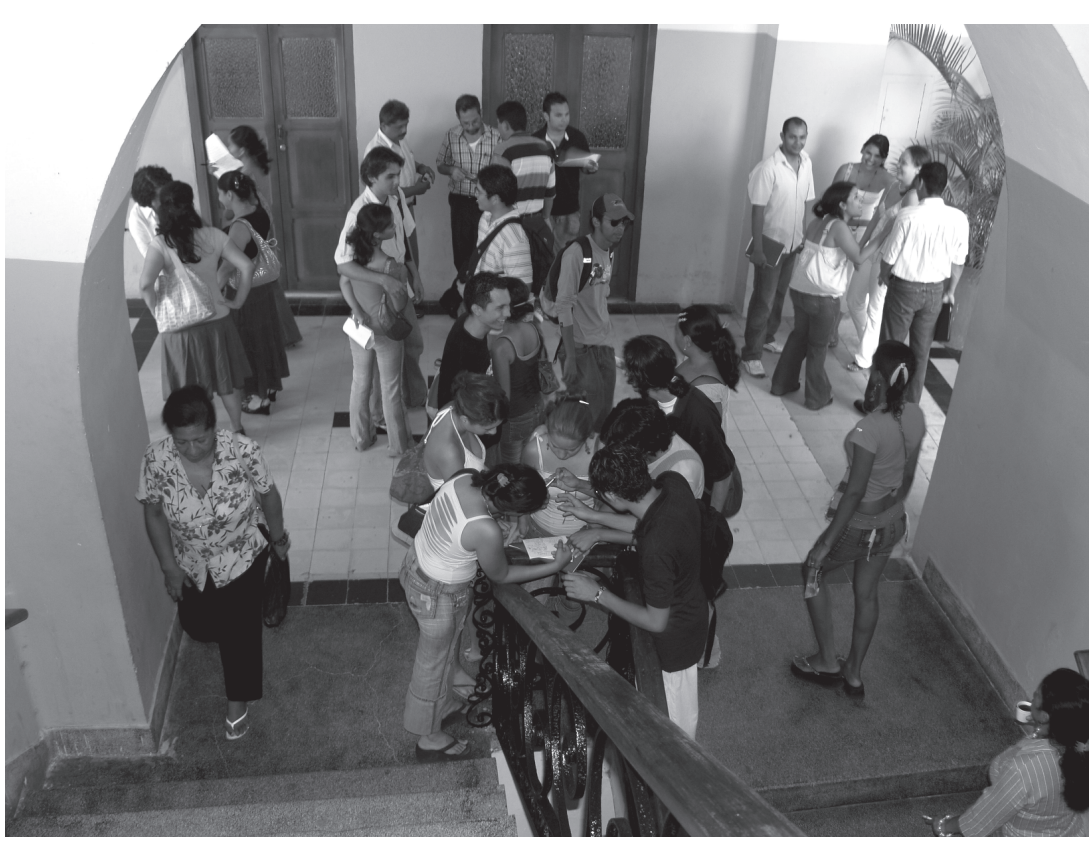

Foto: Mario Lorduy. Sección Publicaciones. Universidad de Cartagena. 
será puesto en su lugar, al servicio de las personas y en consulta con la realidad de la presencia del espíritu que anima la energía en el cosmos fragmentado.

Lo cierto es que por ahora, la propuesta tecnológica surgida de la revolución industrial y la posterior revolución de la informática, han generado en efecto el crecimiento económico sostenido para una pequeña minoría de naciones y habitantes del planeta, a costa de un aumento de los niveles de pobreza para el resto, un estilo de vida fundado en la hipoteca del sistema de crédito, el endeudamiento de las próximas generaciones y del deterioro indiscriminado de los recursos y la salud del planeta.

Los ciudadanos de este mundo tecnológico biotrónico y virtual que se nos pinta como panacea, podrían parecer más máquinas que seres humanos, pues es un mundo en el que la conciencia estorba. Todos estaremos integrados a través de redes de información y de control instaladas desde minúsculos micro-chips bajo nuestra piel y programas de aprendizaje conectados a nuestro cerebro. Ya existen. La pregunta del millón es iqué es lo que estaremos cediendo a cambio de la maravilla tecnológica del mundo virtual?; en otras palabras, ¿en qué consiste la verdadera cuenta de cobro de esta acelerada espiral de velocidad del cambio? ¿Estamos seguros de que ese mundo en el que el valor fundamental es tener y la orden del día es correr para tener, sin importarnos la suerte de los demás, es lo que deseamos para nuestros hijos y nietos?

\section{Un Impacto Inusitado}

Esta primera versión del Festival Ver Ciencia Caribe, culminó su jornada de tres días con un taller de participación pública, en el cual los jóvenes presentaron sus propuestas en torno a la pregunta de cómo pueden ellos participar en la construcción de una cultura científica regional; sus ricos aportes, sistematizados en cerca de ocho categorías, fueron entregados a las instancias de fomento regional y nacional como Colciencias, el Observatorio del Caribe Colombiano y la Asociación Colombiana para el Avance de la Ciencia.

El impacto de la primera versión se evaluó mediante la retroalimentación otorgada por un instrumento aplicado a una muestra de 500 jóvenes estudiantes de secundaria participantes en la emisión televisiva y los talleres del Festival, así como a los orientadores, quienes proporcionaron información sobre eficacia de la estrategia metodológica, cumplimiento de metas y dinámica de participación y motivación de los jóvenes. 
Entre sus sorprendentes resultados, encontramos que el 85\% de los jóvenes entró al Festival con una actitud indiferente o negativa en torno al tema de la ciencia, y al tercer día, un 98\% expresaba una actitud de interés y deseo de continuar formándose en el tema de la ciencia. Este resultado nos sugiere que estamos no sólo ante un modelo de apropiación social del conocimiento científico, sino ante un modelo comunicacional de cambio de actitud altamente exitoso y aplicable a los más diversos ámbitos.

En efecto, los jóvenes proporcionaron información sobre cambios de actitud frente al tema de la relación Ciencia, Tecnología y Sociedad; percepción de aprendizaje sobre temáticas de globalización, ciencia y conectividad, pensamiento crítico y creatividad; y percepción sobre el tratamiento de la información periodística y audiovisual.

La experta internacional en Ciencia, Tecnología, Sociedad e Innovación Elsa Beatriz Acevedo Pineda, quien actuó como asesora externa del proyecto, ha resumido el impacto de Ver Ciencia en seis apreciaciones de valor:

- El proyecto Ver Ciencia Caribe, en el proceso de regionalización de la ciencia y la tecnología, y en materia de apropiación social del conocimiento en una región, no tiene antecedentes en Colombia.

- El modelo por sus características de lectura sociocultural regional, amerita ser apoyado y multiplicado a nivel de otras regiones del país.

- De la misma manera es factible su implementación en países con características similares al nuestro. Es un modelo de Know How especial, en la formación de jóvenes talentos.

- Responde en su totalidad a los patrones de divulgación científica, apropiación social de la ciencia y la tecnología y formación humana integral.

- La entidad que asuma su compromiso, de seguimiento y fortalecimiento, recibe en bandeja de plata el mejor y mayor insumo de endogenización y formación de capital humano para la investigación, el desarrollo y la innovación, conocido hasta ahora.

- Su filosofía, desarrollo y contenidos programáticos, se enmarcan en la formación en valores, ética, responsabilidad y compromiso con el entorno generalizado.

La Habana, Cuba, 6 de Junio de 2006

Palobra No. 7 Agosto/2006 


\section{BIBLIOGRAFÍA}

ACEVEDO, Pineda, Elsa Beatriz. Ciencia y Público. Tomado de Internet

LEON, Bienvenido. (1999) El Documental de Divulgación Científica, Paidós, Papeles de Comunicación 24, Barcelona.

MEJIA, Marco Raúl. (2001) Seminario sobre Pedagogía Social, Universidad de Cartagena. Apuntes.

MEMORIAS DE LA BIENAL IBEROAMERICANA DE LA COMUNICACIÓN: SOCIEDAD, INFORMACIÓN Y CONOCIMIENTO. (2003) Red Latina de Comunicación Social, Universidad de El Salvador. Ponencia de Carlos Ospina Bozzi.

MORAN, Edgar. Los siete saberes necesarios para la educación del futuro. UNESCO- Ministerio de Educación Nacional de Colombia.

OSPINA BOZZI, Carlos y MEJIA, Silvia. (2003) Sincronía en el Espacio Tiempo. Revista Palobra, Universidad de Cartagena, Facultad de Ciencias Sociales y Educación, No, 4.

PLATA, Juan José. Políticas Públicas, conectividad y gestión del conocimiento para la vida. Tomado de Internet.

ROLDAN Castro, Ismael. Humor y creatividad en la divulgación científica desde la televisión: una experiencia persuasiva. Tomado de Internet.

\section{BIOGRAFÍA \\ CARLOS OSPINA BOZZI}

Comunicador Social. Especialista en Administración de Programas de Desarrollo Social Universidad de Cartagena. Docente Facultad de Ciencias Sociales y Educación. Director Proyecto Festival Ver Ciencia Caribe. 